\title{
Spontaneous Orbital Hematoma: A Rare Complication of Vaginal Delivery
}

\author{
Sara Nunes ${ }^{1 *}$ (D) Mariana Guimarães ${ }^{2}$ and Vitor Leal ${ }^{3}$ \\ ${ }^{1}$ Department of Gynecology \& Obstetrics, Centro Hospitalar Trás-os-Montes e Alto Douro, Vila Real, Portugal \\ ${ }^{2}$ Department of Gynecology \& Obstetrics, Centro Hospitalar de São João, Porto, Portugal \\ ${ }^{3}$ Department of Ophtalmology, Centro Hospitalar de São João, Porto, Portugal
}

*Corresponding author: Sara Nunes, Department of Gynecology \& Obstetrics, Centro Hospitalar Trás-os-Montes e Alto Douro, Vila Real, Portugal, Tel: 00351915418111

\begin{abstract}
Background: Nontraumatic orbital hemorrhage is a rare complication after labor with only 12 cases reported in the literature. We describe a new case of a spontaneous, nontraumatic orbital hematoma after an uncomplicated vaginal delivery.

Methods: Retrospective clinical case.

Results: A 34-year-old healthy woman, gesta2 para1, complains, after an uncomplicated, term, vaginal delivery, of left ocular pressure associated with double vision. On physical examination, an orbital asymmetry with left eye proptosis and inferior deviation was observed. Ophthalmological examination revealed a left vertical diplopia with normal visual acuity $(10 / 10$, bilaterally). Pupils were reactive with no papilledema. Orbital CT diagnosed a homogeneous, hyperdense lesion with $6.5 \times 25 \times 34 \mathrm{~mm}$ on the left upper orbit, suggestive of hematoma (Figure 1). Orbital/cranial MRI, performed two days later, confirmed an intraorbital, extra-conic acute hematoma with $7 \mathrm{~mm}$ (maximum diameter), imposing inferior deviation of left ocular globe. Expectant management was adopted and one month after all symptoms and signs had disappeared.
\end{abstract}

Conclusions: Complete regression of orbital hematoma with conservative treatment strongly supports the diagnosis of nontraumatic orbital hemorrhage.

\section{Introduction}

Nontraumatic orbital hemorrhage is an uncommon clinical entity, usually associated with vascular anomalies in the elderly [1]. It is even a rarer complication after labor with only 12 cases reported in the literature [2]. It seems to be associated with increased orbital vascular pressure during maternal expulsive efforts $[2,3]$.

Though the relationship of orbital hemorrhage after Valsalva Maneuver is well known, it is important to bring awareness of this pathology to healthcare professionals, especially to those who provide obstetric care, since visual complains may be associated with severe obstetric complications such as pre-eclampsia/ eclampsia, which should be excluded. We present a new case of a spontaneous, orbital hematoma on a healthy woman, after an uncomplicated, term, vaginal delivery.

\section{Case Description}

A 34-year-old healthy woman, gesta2 para1, was admitted, at term (39 weeks and 4 days) in our obstetric unit on spontaneous labor. There were no reported complications during the pregnancy and labor developed uneventfully, with normal duration and evolution. Vaginal delivery of a female newborn with normal weight (3160 grams) occurred without need of medical instrumentation. During final expulsive efforts, the mother complained of left ocular pressure and, after delivery, the feeling of double vision. On physical examination, she had an orbital asymmetry with left eye proptosis and inferior deviation. Ophthalmological examination revealed a left vertical diplopia with normal visual acuity (10/10, bilaterally). Pupils were reactive and there was no papilledema. Orbital CT diagnosed a homogeneous, hyperdense lesion with $6.5 \times 25 \times 34 \mathrm{~mm}$ on the left upper orbit, suggestive of hematoma (Figure 1). Orbital/cranial MRI, performed 


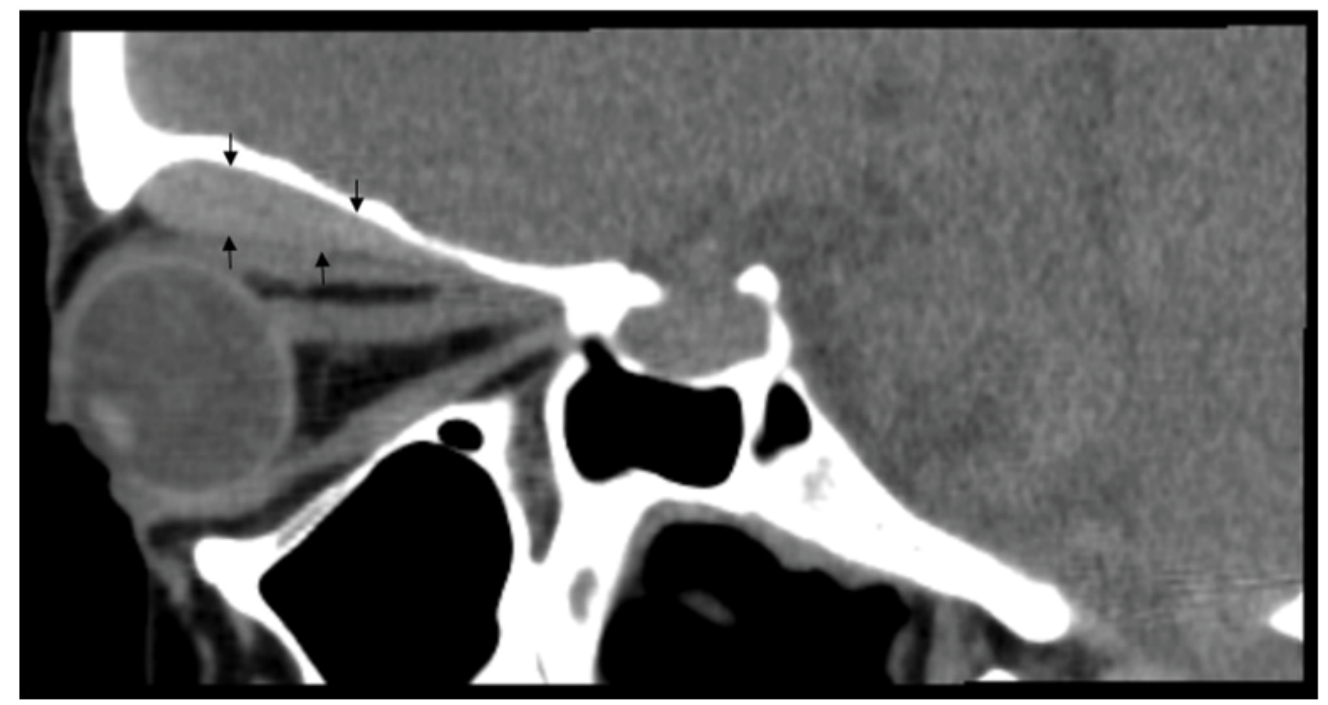

Figure 1: Hematoma on the left upper orbit (black arrows) on orbital CT.

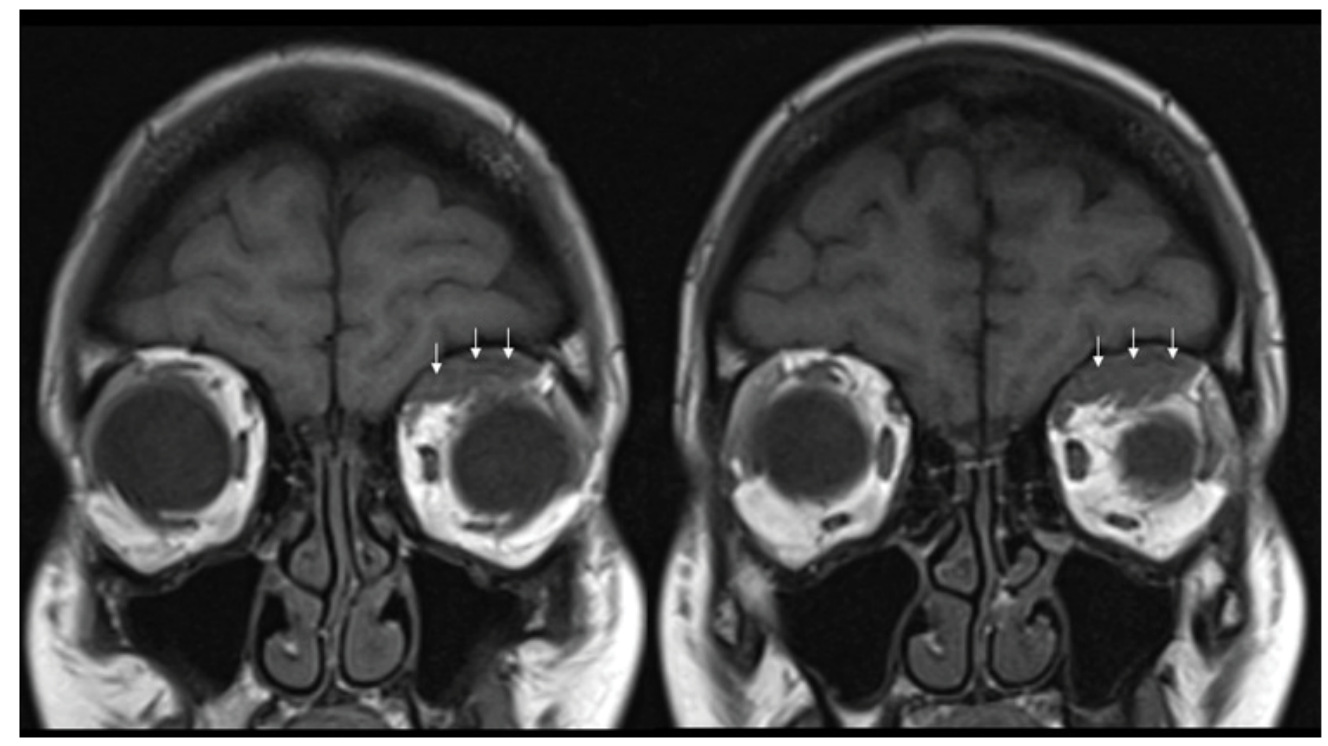

Figure 2: Hematoma on the left upper orbit (white arrows) on cranial MRI.

two days later, confirmed an intraorbital, extra-conic acute hematoma with $7 \mathrm{~mm}$ (maximum diameter), imposing inferior deviation of left ocular globe (Figure 2). Expectant management was adopted and no other clinical complains were registered.

One month after hospital discharge, all ophthalmologic symptoms and signs had disappeared, which strongly supports the diagnosis of nontraumatic orbital hemorrhage.

\section{Conclusion}

Spontaneous orbital hemorrhage is a rare complication after vaginal delivery, and it usually develops without early warning. Healthcare providers should be alert to this entity and other obstetric pathologies that present with visual symptoms, such pre-eclampsia/eclampsia, must be excluded. Despite causing great concern to patients, it usually presents good prognosis with conservative treatment.

\section{Sources of Support}

None.

\section{Conflicts of Interest Statement}

None of the authors have conflict of interest to declare and all presented equal contribution for this article.

\section{References}

1. Paramanathan V, Zolnourian A (2011) Spontaneous intraorbital hematoma: Case report. Clinical Ophthalmology 5: $1-2$.

2. Riri K, Zacharis K, Tsironi E, Daponte A, Papageorgiou E (2021) Subperiosteal orbital hemorrhage: An unusual complication during labor. J Craniofac Sur 32: e219-e220.

3. Oruç S, Sener E, Akman A, Sanaç A (2001) Bilateral orbital hemorrhage induced by labor. European Journal of Ophthalmology 11: 77-79. 